\title{
Functional connectivity between brain regions involved in learning words of a new language
}

\author{
Kim Veroude ${ }^{a}$, David G. Norris ${ }^{a}$, Elena Shumskaya ${ }^{a}$, Marianne Gullberg ${ }^{b}$, Peter Indefrey ${ }^{\text {a,b,c,* }}$ \\ ${ }^{a}$ Radboud University Nijmegen, Donders Institute for Brain, Cognition and Behaviour, P.O. Box 9101/204, NL-6500 HB Nijmegen, The Netherlands \\ ${ }^{\mathrm{b}}$ Max Planck Institute for Psycholinguistics, P.O. Box 310, NL-6500 AH Nijmegen, The Netherlands \\ ${ }^{\mathrm{c}}$ Heinrich Heine University Düsseldorf, Institut für Sprache und Information, Universitätsstr. 1, D-40225 Düsseldorf, Germany
}

\section{A R T I C L E I N F O}

\section{Article history:}

Accepted 18 December 2009

Available online 29 January 2010

\section{Keywords:}

Language

Word learning

Second language

Phonology

Functional connectivity

Resting state

\begin{abstract}
A B S T R A C T
Previous studies have identified several brain regions that appear to be involved in the acquisition of novel word forms. Standard word-by-word presentation is often used although exposure to a new language normally occurs in a natural, real world situation. In the current experiment we investigated naturalistic language exposure and applied a model-free analysis for hemodynamic-response data. Functional connectivity, temporal correlations between hemodynamic activity of different areas, was assessed during rest before and after presentation of a movie of a weather report in Mandarin Chinese to Dutch participants. We hypothesized that learning of novel words might be associated with stronger functional connectivity of regions that are involved in phonological processing. Participants were divided into two groups, learners and non-learners, based on the scores on a post hoc word recognition task. The learners were able to recognize Chinese target words from the weather report, while the non-learners were not. In the first resting state period, before presentation of the movie, stronger functional connectivity was observed for the learners compared to the non-learners between the left supplementary motor area and the left precentral gyrus as well as the left insula and the left rolandic operculum, regions that are important for phonological rehearsal. After exposure to the weather report, functional connectivity between the left and right supramarginal gyrus was stronger for learners than for non-learners. This is consistent with a role of the left supramarginal gyrus in the storage of phonological forms. These results suggest both pre-existing and learning-induced differences between the two groups.
\end{abstract}

(c) 2010 Elsevier Inc. All rights reserved.

\section{Introduction}

Vocabulary acquisition is an important part of learning a new language. One aspect of this process is remembering and recognizing phonological information about novel word forms. There is considerable evidence that the phonological loop, a component of working memory, plays a role in acquiring novel word forms (Baddeley, 1986; Baddeley, Gathercole, \& Papagno, 1998). The phonological loop consists of a subvocal rehearsal system and a phonological store. Neuroimaging research using positron emission tomography (PET) and functional magnetic resonance imaging (fMRI) suggests that the pars opercularis (Brodmann area (BA) 44 , part of Broca's area) and the left supplementary motor area (BA 6) are involved in phonological rehearsal while the left supramarginal gyrus (BA 40) mediates the storage of phonological forms (Paulesu, Frith, \& Frackowiak, 1993; Smith, Jonides, Marshuetz, \& Koeppe, 1998).

\footnotetext{
* Corresponding author. Address: Institut für Sprache und Information, Heinrich Heine University Düsseldorf, D-40225 Düsseldorf, Germany.

E-mail address: indefrey@phil-fak.uni-duesseldorf.de (P. Indefrey).
}

Indeed, several studies have found these brain regions to be involved in word learning. The magnitude of activation in left inferior prefrontal cortex and bilateral parietal regions has been shown to be greater for new than for familiar words and positively correlated with memory for these items (Clark \& Wagner, 2003). Breitenstein et al. (2005) demonstrated increased activity in the left supramarginal gyrus during learning of a novel lexicon, in addition to modulations of the left hippocampus and fusiform gyrus. A positive correlation between vocabulary knowledge and gray matter density in bilateral supramarginal gyri has also been observed (Lee et al., 2007).

An involvement of inferior parietal cortex in lexical learning has also been found with magnetoencephalography (MEG). Cornelissen et al. (2004) showed that learning names for new objects was associated with a source in the inferior parietal lobe in four out of five subjects. However, a PET experiment that compared naming of novel and familiar objects, similar to those used by Cornelissen et al. (2004), did not find inferior parietal cortex involvement but regional cerebral blood flow ( $\mathrm{rCBF}$ ) increases in the left inferior frontal cortex, the left anterior temporal area and the cerebellum (Gronholm, Rinne, Vorobyev, \& Laine, 2005). The right cerebellum, the left insula 
and right medial temporal regions were also related to second language vocabulary learning in another PET study, which in addition demonstrated increased activity in the left posterior superior temporal sulcus and the middle temporal cortex for subjects with good memory performance on new words (Raboyeau et al., 2004). The left insula has further been shown to play a role in phonological recognition of words (Bamiou, Musiek, \& Luxon, 2003). On a phonological working memory task, increasing load resulted in increased activation in the middle frontal gyrus, the insula, superior temporal gyrus and inferior parietal cortex. Of these areas, the left insula has been found to be more strongly activated in second language processing for balanced compared to unbalanced bilinguals (Chee, Soon, Lee, \& Pallier, 2004).

Given that vocabulary learning studies have used different training regimes ranging from hours to days of training, it is not clear at which point in time the observed neural differences between novel and familiar words may arise. Recently, Davis, Di Betta, Macdonald, and Gaskell (2008) investigated the behavioral and neurocognitive correlates of novel word form learning on two successive days. They found a behavioral effect (lexical competition of novel word forms with existing words) and stronger neocortical hemodynamic activation for novel word forms (left superior temporal, left inferior frontal, and premotor regions) to depend on overnight consolidation. In contrast, enhanced hippocampal activation was observed for novel word forms learned on the same day.

Finally, neural correlates of differences in learning success have also been found before participants were exposed to novel words. For example, Wong, Perrachione, and Parrish (2007) investigated individual differences in the learning of lexical pitch patterns. They found stronger activation in the left posterior superior temporal region for successful learners while less successful learners displayed increased activation in areas involved in nonlinguistic pitch processing, working memory and attention. The activity in the superior temporal cortex differed between the two groups even before language training. Mei et al. (2008) studied the learning of word forms of an unknown language that were presented both visually and auditorily. These authors also observed pre-existing differences between good and poor learners with the former having more activation in left middle temporal gyrus and superior temporal sulcus and less activation in right inferior frontal gyrus.

In summary, brain regions that appear to be involved in phonological acquisition of new vocabulary are the left supramarginal gyrus, suggested to function as a neural store for novel sound forms, the pars opercularis and left supplementary motor area suggested to be involved in subvocal rehearsal, as well as the left insula and left posterior superior and middle temporal gyri that have often been found in research investigating phonological learning.

Previous studies on vocabulary acquisition have used standard word-by-word presentation (Breitenstein \& Knecht, 2002; Breitenstein et al., 2005; Mei et al., 2008; Wong et al., 2007). However, exposure to an unknown language usually occurs in a complex real world situation where words occur in a continuous speech stream. A recent study investigated the behavioral effects of naturalistic exposure to a new language (Gullberg, Roberts, \& Dimroth, in press). Dutch native speakers were presented with a movie of a weather report in Mandarin Chinese, a typologically unrelated language that was unknown to the participants. Afterwards, an auditory word recognition task had to be performed. The subjects were able to recognize words from the weather report that had occurred frequently and were highlighted by a gesture. Mean accuracy for these words was $64 \%$ (with a $50 \%$ chance level) after one exposure to the movie (6:14 $\mathrm{min})$ and increased to $71 \%$ after two exposures. These results show that acoustic or phonological units can be segmented from the continuous speech stream during minimal exposure to a new language. In the current fMRI experiment, we use the materials from this study and apply a data-driven analysis method. This might provide additional information on aspects of vocabulary learning in a naturalistic context.

Traditionally, hemodynamic-response data are analyzed by contrasting a condition of interest with a control condition, such as a lower-level baseline or rest. A blocked or event-related design specifying the time course of the conditions is used and the General Linear Model (GLM), a statistical model that takes into account the temporal order and duration of conditions, is applied. An increase in neural activity, measured indirectly as an increase in blood flow, indicates that a region is involved in the experimental task. Given that in the case of natural stimuli there is no pre-specified experimental design, methods that complement the GLM are needed. One possibility is to look at functional connectivity, correlations between the neurophysiological activation time courses of spatially distinct areas, which constitutes a model-free approach (Friston, Frith, Liddle, \& Frackowiak, 1993). In contrast, effective connectivity, the influence of one neural system on another, requires a priori specification of connections of interest.

During rest, functionally related brain regions display correlations in the fMRI time courses. This was first demonstrated in the motor system (Biswal, Yetkin, Haughton, \& Hyde, 1995). Functional connectivity has also been shown between other areas, for instance in the visual cortex (Lowe, Mock, \& Sorenson, 1998), the language system (Cordes et al., 2000) and in the so-called default mode network (Greicius, Krasnow, Reiss, \& Menon, 2003), a set of regions that show deactivations during task performance (Raichle et al., 2001). Recently, Damoiseaux et al. (2006) identified 10 functionally relevant resting state networks by Independent Component Analysis (ICA) which were found to be consistent across subjects and across sessions. These findings suggest that functional connectivity reflects true interaction between brain regions, although there is also some evidence for an effect of physiological noise, such as low frequency changes in respiration (Birn, Diamond, Smith, \& Bandettini, 2006).

Other researchers have demonstrated that depending on the state of the brain, the strengths of correlations in functional networks can differ. In patients suffering from schizophrenia, decreases in connectivity have been observed which may reflect disrupted functional integration in the brain (Liang et al., 2006). Altered functional connectivity is present in other disorders as well, including autism (Cherkassky, Kana, Keller, \& Just, 2006) and ADHD (Tian et al., 2006). More subtle, temporary changes in correlations between brain regions also occur and can be the result of task performance. For example, functional connectivity between Broca's area and Wernicke's area was observed during rest and increased during continuous listening (Hampson, Peterson, Skudlarski, Gatenby, \& Gore, 2002). In resting state after performance of a language task, stronger correlations were found between regions involved in that task (Waites, Stanislavsky, Abbott, \& Jackson, 2005).

Functional and effective connectivity have been related to associative learning. In an experiment by Büchel, Coull, and Friston (1999), subjects performed a task in which line drawings of objects were paired with locations. Over time, activation decreased in the ventral and dorsal visual stream, which indicates repetition suppression. In contrast, effective connectivity between these areas increased. These changes were correlated with individual task performance which shows that interactions between brain regions play a role in learning. Others have demonstrated comparable findings (McIntosh, Rajah, \& Lobaugh, 1999; Toni, Rowe, Stephan, \& Passingham, 2002).

In the current exploratory study we investigate differences in functional connectivity related to the learning of novel words. We focus on six regions known to be involved in phonological processing, namely the pars opercularis, the left supplementary motor area, the left insula, the left supramarginal gyrus, the left posterior supe- 
rior temporal gyrus and left posterior middle temporal gyrus. We propose that functional connectivity of these areas is related to the learning of new word forms. More specifically, the recruitment of a phonological network might manifest itself as stronger correlations between regions reflecting interactive processing. Therefore, we predict stronger functional connectivity for subjects that are able to extract phonological word form information from a continuous speech stream compared to subjects that do not learn novel words.

\section{Methods}

\subsection{Participants}

Thirty-four healthy volunteers participated in the study (22 females, mean age 22 , range 18-24) after giving written informed consent. They received financial compensation or course credit for their collaboration. All were native Dutch speakers without any knowledge of Chinese or a related language, according to their answers on a language background questionnaire (Gullberg \& Indefrey, 2003). The participants were right-handed, had normal or corrected-to-normal vision and no hearing problems.

\subsection{Materials}

We used a movie of a naturalistic but fully controlled weather report in Mandarin Chinese, showing a news reader speaking and gesturing over weather charts (Gullberg et al., in press; for an example see Supplementary materials). Six weather charts were presented with a verbal description consisting of continuous speech by a native Mandarin Chinese speaker. The movie lasted 6:14 min and contained 24 target words in four conditions of six target words (two verbs, two nouns, two function words) each: frequent (eight occurrences) or infrequent (two occurrences) and highlighted (yes or no) by a gesture forming a deictic link to the referential context, the icons on the weather chart. In a subsequent auditory word recognition task the 24 target words and 72 filler words, matched for phonological syllable structure, were presented randomly. Participants had to indicate by button press whether they had heard the word in the weather report or not. Two versions of this task were created using Presentation software (www.neurobs.com) and counterbalanced across subjects to avoid order effects.

\subsection{Experimental paradigm}

The fMRI session consisted of five parts. First, there was a resting state period of $5 \mathrm{~min}$, followed by the first presentation of the weather report, then another resting state period, the second presentation of the weather report and finally a third resting state period. Before each part, the instructions were presented onto a backprojection screen that could be seen through a tilted mirror. Participants were instructed to close their eyes and relax during rest and to attentively watch and listen to the movie. They were also informed that after the fMRI session there would be two further tasks and they would be asked to fill in a questionnaire. A piece of music was played after 5 min of rest which indicated that the subjects could open their eyes. An eye tracker was used to verify that the eyes were closed during rest and open during presentation of the weather report. The sound level was adjusted for each participant individually at the start of the experiment to make sure they could hear the movie against the background scanner noise. After the fMRI session, subjects performed an auditory word recognition task for which they indicated by button press whether they had heard the word in the weather report or not and a six alternative forced choice semantic decision task in which they were asked to indicate the correct meaning of words they had heard during the weather report.

\section{4. fMRI data analysis}

The fMRI data were acquired on a $3 \mathrm{~T}$ Trio scanner (Siemens, Erlangen, Germany). We scanned continuously using a functional T2* weighted EPI-BOLD sequence with the following parameters: $\mathrm{TE}=30 \mathrm{~ms}, \mathrm{TR}=1350 \mathrm{~ms}$, flip angle $=67^{\circ}$, number of slices $=21$, interleaved acquisition order, voxel size $=3.5 \times 3.5 \times 4.0$, bandwidth $=1906 \mathrm{~Hz} /$ pixel. Preprocessing of the functional data was carried out using SPM5 (www.fil.ion.ucl.ac.uk/spm). The images were realigned and normalized to the Montreal Neurological Institute (MNI) EPI template. Following the procedures of Salvador et al. (2005) no slice time correction and no spatial smoothing were performed. Temporal filtering was applied by using a band pass Butterworth filter with frequency borders between 0.01 and $0.08 \mathrm{~Hz}$

We used a resting state toolbox (in-house software) in MATLAB (The MathWorks, Inc., Natick, MA) to carry out a whole brain partial correlation analysis based on the method of Salvador et al. (2005). This provides unique correlation coefficient values between pairs of regions with contributions of other areas and autocorrelation cancelled out. The regions that were included in the analysis were defined by the AAL template, consisting of 116 areas (Tzourio-Mazoyer et al., 2002). For our purposes, the superior and middle temporal gyri in both hemispheres were divided into a posterior third and an anterior two-thirds. This was done because the posterior parts of these areas in the left hemisphere are known to play an important role in receptive language processing. Hence, the template we used contained 120 regions and we assessed partial correlations between the six regions of interest and all other areas. Our regions of interest were the following: the pars opercularis of the left inferior frontal gyrus, the left supplementary motor area, the left insula, the left supramarginal gyrus, the left posterior superior temporal gyrus and the left posterior middle temporal gyrus.

Starting with the fourth volume acquisition after the end of the movie clip, the time courses of each region in the template during the first, second and third resting state period and the two weather report presentation periods were extracted for every subject. Based on this, a sample covariance matrix was constructed which was rescaled to form a partial correlation matrix. Fisher's $r$ to $z$ transformation was carried out to improve normality. Three thousand four hundred and ninety-five one sample $t$-tests $(p<0.05$, FDR corrected) were performed to identify partial correlations between any of the six regions of interest and any other region (699 region pairs $* 5$ periods) that were significantly different from zero. For ten region pairs showing significant partial correlations in the last resting state period, further tests were conducted.

An overall mixed ANOVA ( $p<0.05)$ with within-subject factors region pair (1-10), resting state period $(1,2,3)$ and between-subject factor group (learners, non-learners) was conducted to test for an interaction effect. For all region pairs and resting state periods, post hoc one-sided independent samples $t$-tests $(p<0.05$, Bonferroni corrected for multiple comparisons) were carried out to see whether, according to our prediction, learners showed stronger functional connectivity compared to non-learners. Because of the exploratory nature of our study, in addition we report partial correlations at a more lenient threshold to reduce the risk of false negatives and to generate hypotheses about areas whose functional connectivity needs to be investigated in further studies.

\section{Results}

\subsection{Behavioral results}

Performance on the word recognition task was assessed using the $D$-prime measure of signal detection theory, which takes 
response bias into account by calculating the difference between the normalized hit and false alarm rate (Macmillan \& Creelman, 2005). Based on their performance on the word recognition task participants were divided into two groups. Subjects with a $D$-prime higher than 0.3 showed some sensitivity to the difference between previously heard Chinese words and new Chinese words and were included in the learners group $(N=14$, range $0.32-0.88$, mean 0.52 , SD 0.16). Participants with a $D$-prime between -0.3 and 0.3 formed the non-learners group ( $N=17$, range -0.28 to 0.22 , mean -0.03 , SD 0.18 ) because their scores indicated that they did not distinguish between previously heard and new words. Three subjects were excluded, because their $D$-prime was below -0.3 , suggesting that they were sensitive to the previously heard words but for unknown reasons rejected them on the task.

Performance on the semantic decision task was at chance level for both groups.

\section{2. fMRI results}

The analysis of the partial correlation matrix yielded ten region pairs with partial correlations that were significantly different from zero during the third resting state period. Table 1 shows the mean $z$-transformed partial correlation coefficients of these region pairs for all resting state periods and the two weather report presentations.

Results of the overall mixed ANOVA with within-subject factors region pair (1-10), period $(1,2,3)$ and the between-subject factor group (learners, non-learners) showed a significant main effect of region $(F(9,261)=11.09, p<0.000)$ reflecting differences in connectivity strength between region pairs and a significant region pair $*$ period $*$ group interaction $(F(18,522)=4.26, p<0.01)$ showing that functional connection strength was not the same for learners and non-learners in all resting state periods and region pairs.

Bonferroni corrected post hoc tests revealed that during the first resting state period, the partial correlation between the left supplementary motor area and the left precentral region was significantly higher for the learners than for the non-learners (mean learners $=0.29$, mean non-learners $=0.09, t(29)=3.38, p=0.03$ ). At a less conservative uncorrected threshold, there were also stronger partial correlations for the learner group compared to the non-learner group between the left insula and the left rolandic operculum during the first resting state period (mean learners $=0.28$, mean non-learners $=0.11, t(29)=2.63, p<0.007$, uncorrected) and between the left and right supramarginal gyri, during the third resting state period (mean learners $=0.55$, mean non-learners $=0.41$, $t(29)=1.81, p=0.04$, uncorrected, see Figs. 1 and 2). No group differences between learners and non-learners were found during the second resting state period.

\section{Discussion}

In this exploratory study we investigated functional connectivity before, between, and after first exposure to an unknown language in a quasi-naturalistic context, a Chinese weather report. Based on previous studies showing changes in functional connectivity that were positively correlated with task performance (Büchel et al., 1999) and task-related changes in resting state connectivity (Waites et al., 2005), we expected stronger resting state connectivity between regions involved in phonological processing for participants who were able to segment possible word forms from the continuous Chinese speech stream and subsequently recognize them, compared to participants who were unable to do so.

A partial correlation analysis was performed for six regions of interest that are known to play a role in phonological processing, namely the left inferior frontal opercular region, the left supplementary motor area, the left insula, the left supramarginal gyrus, the left posterior superior temporal gyrus and the left posterior middle temporal gyrus. Confirming the findings of Salvador et al. (2005) and Stark et al. (2008), we observed large differences in connectivity strength between regions, with the strongest functional connectivity between homologous regions of the left and right hemisphere. Intrahemispheric functional connections involving our regions of interest were only found with neighboring areas, thus confirming the effect of anatomical distance reported by Salvador et al. (2005). Furthermore, intrahemispheric functional connections tended to be weaker (with the exception of the pars opercularis and the pars triangularis of the left inferior frontal gyrus) and less reliable across measurement periods. Among the interhemispheric connections those between motor-related regions (SMA, insula) tended to be stronger than those between temporal lobe regions. Unlike the previous studies, we found the left and right supramarginal gyri to show similar functional connectivity strength as the motor-related regions with strongest functional connectivity during the last resting state period.

Partial correlations during presentation of the weather report are not directly comparable to resting state partial correlations, because they can be driven to an unknown extent by hemodynamic stimulus responses. Nonetheless, our results suggest that the region pairs showing the strongest connectivity during the resting state periods (interhemispheric SMA, insula, and supramarginal gyrus connections) were even more strongly connected while participants processed the weather report.

While our results confirm that differences in resting state functional connectivity between regions reflect largely interhemispheric and local anatomical connectivity, we also observed that the functional connectivity between some regions was further modulated by word recognition success. During the first resting

Table 1

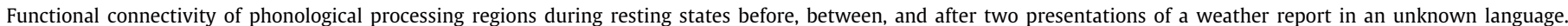

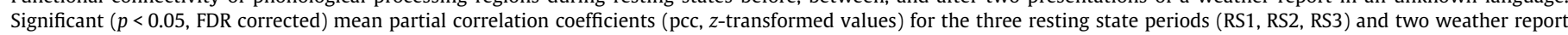

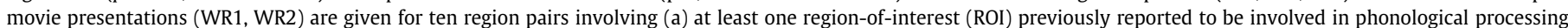

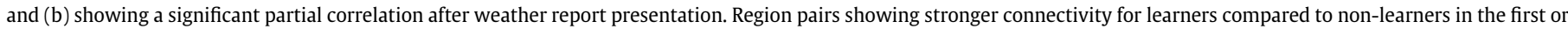

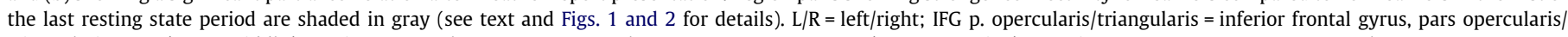
triangularis; MTG/STG = middle/superior temporal gyrus; SMA = supplementary motor area; ant. $/$ post. = anterior/ posterior; gyr. = gyrus; operc. = operculum.

\begin{tabular}{|c|c|c|c|c|c|c|}
\hline Region 1 & Region 2 & RS 1 & RS 2 & RS 3 & WR 1 & WR 2 \\
\hline L. IFG p. opercularis & L. IFG, p. triangularis & 0.31 & 0.28 & 0.30 & 0.46 & n.s. \\
\hline L. SMA & L. precentral gyrus & 0.18 & n.s. & 0.21 & n.s. & n.s. \\
\hline L. SMA & R. SMA & 0.38 & 0.40 & 0.39 & 0.44 & 0.51 \\
\hline L. insula & L. IFG, p. orbitalis & 0.06 & n.s. & 0.18 & n.s. & n.s. \\
\hline L. insula & L. rolandic opercul. & 0.18 & n.s. & 0.20 & 0.26 & n.s. \\
\hline L. insula & $\mathrm{R}$. insula & 0.31 & 0.29 & 0.32 & 0.40 & 0.49 \\
\hline L. supramarginal gyr. & R. supramarginal gyr. & 0.40 & 0.35 & 0.48 & 0.38 & 0.51 \\
\hline L. post. STG & R. post. STG & 0.30 & 0.29 & 0.32 & n.s. & 0.29 \\
\hline L. post. MTG & L. ant. MTG & 0.23 & 0.25 & 0.22 & n.s. & 0.37 \\
\hline L. post. MTG & R. post. MTG & 0.31 & 0.28 & 0.33 & 0.31 & n.s. \\
\hline
\end{tabular}




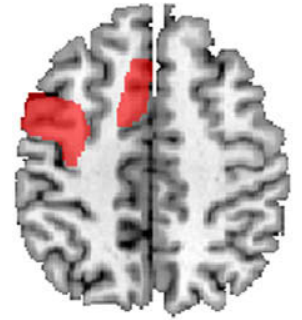

anorlearners a learners

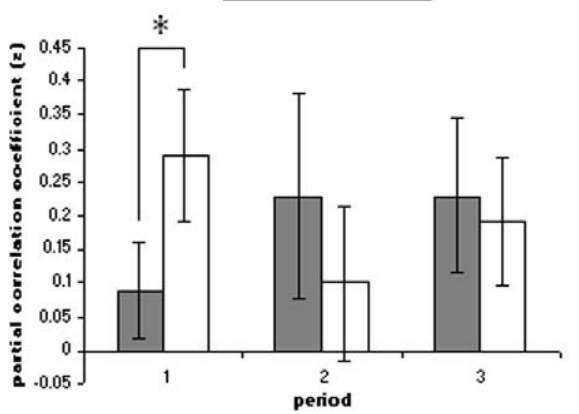

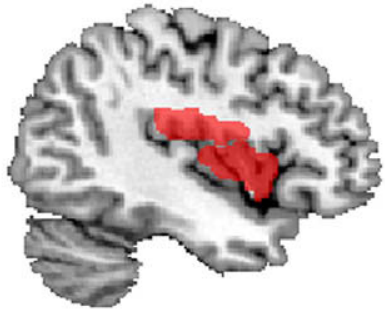

a nontearners a learners

(*)

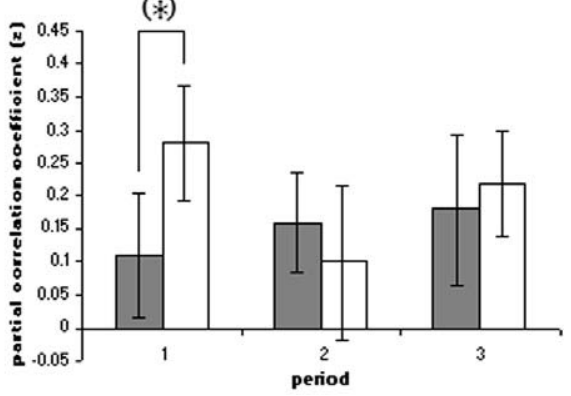

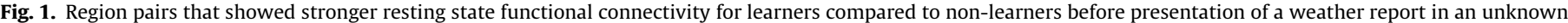

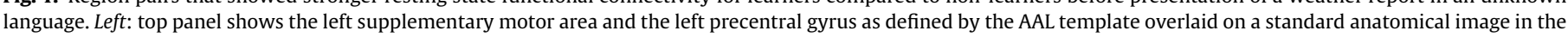

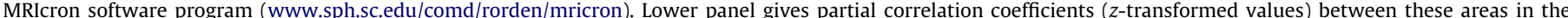

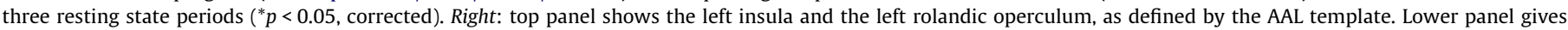
partial correlation coefficients for these areas in the three resting state periods $\left({ }^{(*)} p<0.05\right.$, uncorrected). Error bars \pm 2 SE are shown.
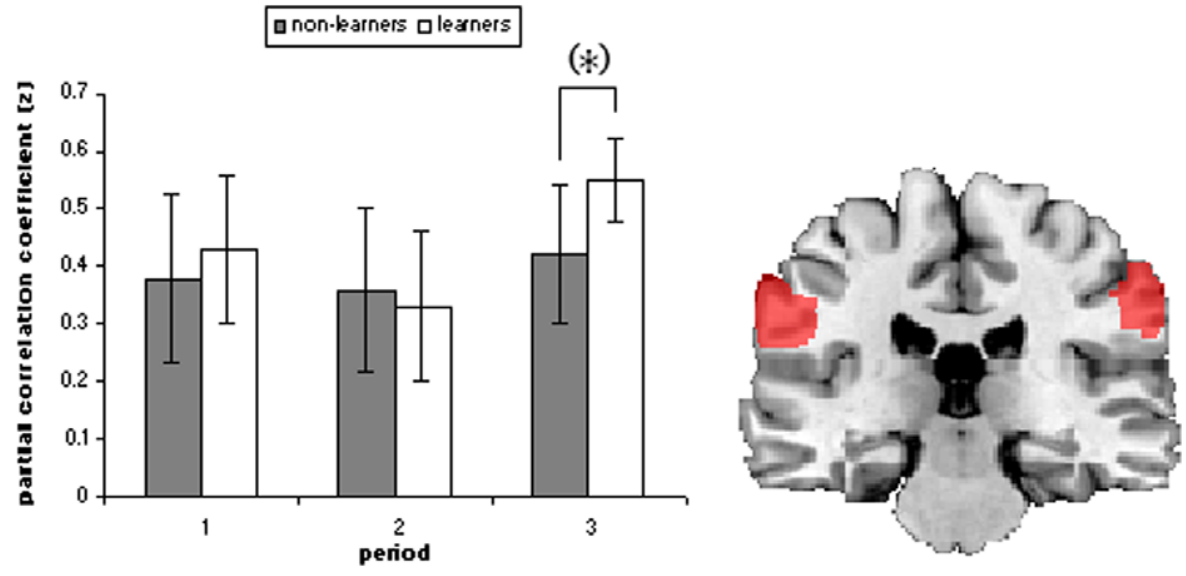

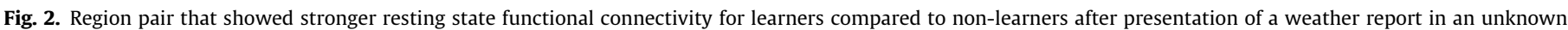

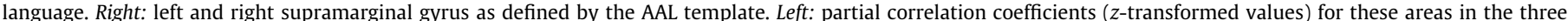
resting state periods $\left({ }^{(*)} p<0.05\right.$, uncorrected). Error bars \pm 2 SE are shown.

state period, the left supplementary motor area and the left precentral gyrus showed stronger functional connectivity for learners compared to non-learners. The left supplementary motor area is important for phonological rehearsal (Paulesu et al., 1993; Smith et al., 1998) and the left precentral gyrus is involved in articulation of speech. A lesion of this area may produce Broca's aphasia (Mori, Yamadori, \& Furumoto, 1989).

Stronger functional connectivity for learners was also observed at a more lenient uncorrected statistical threshold between the left insula and the left rolandic operculum. The left rolandic operculum plays a role in speech production, as demonstrated by the finding that lesions of this area are associated with articulatory disorders (Tonkonogy \& Goodglass, 1981). The left insula appears to contribute to planning (Dronkers, 1996) or execution of speech (Ackermann \& Riecker, 2004). An involvement of the left insula in phonological recognition of words has also been previously shown
(Bamiou et al., 2003). Using a phonological working memory task, Chee et al. (2004) found greater activation of this region for balanced than for unbalanced bilinguals.

In sum, although these areas have multiple functions, with respect to language they seem to be predominantly involved in articulation and phonological rehearsal. One possible interpretation might thus be that learners and non-learners differed with respect to the involvement of the speech motor system during perception. However, these regions were not significantly functionally connected during the weather report presentations and showed a dissociation between learners and non-learners only before presentation of the Chinese weather report. Stronger functional connectivity between these areas, and hence possibly an increased efficiency of speech motor activation, may thus rather be considered a favorable disposition for the processing of the unknown language input. 
One pair of regions, the left and right supramarginal gyrus, showed stronger functional connectivity at an uncorrected statistical threshold during the last resting state period for the learners compared to the non-learners. The correlation between these areas was similar for the two groups during the first and second resting state periods. This suggests that there were no pre-existing differences between the learners and non-learners with respect to this region pair, so the stronger functional connectivity is related to exposure to the weather report movie.

In line with Waites et al. (2005), we interpret stronger functional connectivity after exposure as being due to an involvement of the supramarginal gyri in stimulus processing during exposure. This assumption is further supported by the increase in functional connectivity between the left and right supramarginal gyri observed for both learners and non-learners during the weather report presentations. Given that participants did not extract any meaning for the word forms they recognized, it is unlikely that the Chinese words were processed beyond a phonological representation. Our finding is consistent with the suggested role of the left supramarginal gyrus as a phonological working memory store (Paulesu et al., 1993; Smith et al., 1998) but could reflect other aspects of phonological working memory as well (see Buchsbaum \& D'Esposito, 2008, for a criticism of the assignment of the phonological storage function to a specific region).

Our results on connectivity patterns before, during, and after exposure to a natural continuous language confirm and complement findings from a quite different, artificial-language paradigm that has been used to study the ability of listeners to segment "words" out of streams of CV syllables based on statistical cues (i.e. different frequencies of syllable co-occurrence; Saffran, Aslin, \& Newport, 1996). In an fMRI study, McNealy, Mazziotta, and Dapretto (2006) found that hemodynamic activation in bilateral posterior temporal and inferior parietal regions including the supramarginal gyri increased more strongly as a function of exposure duration when the language stream provided cues to word segmentation than when it did not. Other areas, including bilateral inferior frontal gyri, precentral gyri, and insulae, were found to be active during exposure but did not show an activation increase over time. The authors interpret activation of the left supramarginal gyrus as reflecting "the development of phonological representations for the "words" in the artificial languages". The stronger connectivity between this area and its right-hemispheric homolog we observed for learners compared to non-learners is in line with this interpretation. More in general, the parallel findings of our study provide some evidence that the artificial language segmentation paradigm captures important aspects of natural language learning.

We observed considerable inter-subject variability in partial correlation values as evident by the large standard deviations in our data, probably precluding the detection of more significant changes in connectivity strength. Therefore, absence of differences in functional connectivity should be interpreted with caution. Our data certainly do not exclude that other areas are also involved in learning novel words. Another caveat to note here is that our behavioral scores were lower than those observed by Gullberg et al. (in press), that is, our results give a too pessimistic view of how much can be learned during first exposure to a new language. Consequently, learners and non-learners' scores on the word recognition test were not well separated, thus reducing the possibility to observe learning-related group differences in functional connectivity. Although we ensured that the participants were able to hear the movie sound, it is likely that there was some interference from the scanner noise. Our results demonstrate the feasibility to study word learning from naturalistic language input in the scanner but future studies need to optimize learning conditions for such stimuli.
To conclude, the current study provides some evidence for differences in functional connectivity between learners and nonlearners of word forms of an unknown language. Additional research is needed to elucidate the effect of task-related activity on functional connectivity during rest. Nonetheless, it was shown here that investigating functional connectivity between different regions can be a useful method when there is no pre-specified design for an experiment, as in the case of naturalistic stimuli. Datadriven techniques provide additional information on how brain regions work together and can therefore complement traditional fMRI research.

\section{Acknowledgments}

The authors would like to thank Paul Gaalman for assistance during fMRI scanning and Frauke Hellwig for help with the materials. This research was partially funded by a Grant from the Nederlandse Organisatie voor Wetenschappelijk Onderzoek, NWO, MPI 56-384, The dynamics of multilingual processing, awarded to $\mathrm{M}$. Gullberg, P. Indefrey, and W. Klein.

\section{Appendix A. Supplementary data}

Supplementary data associated with this article can be found, in the online version, at doi:10.1016/j.bandl.2009.12.005.

\section{References}

Ackermann, H., \& Riecker, A. (2004). The contribution of the insula to motor aspects of speech production: A review and a hypothesis. Brain and Language, 89(2), 320-328.

Baddeley, A., Gathercole, S., \& Papagno, C. (1998). The phonological loop as a language learning device. Psychological Review, 105(1), 158-173.

Baddeley, A. D. (1986). Working memory. Oxford, England: Oxford University Press.

Bamiou, D. E., Musiek, F. E., \& Luxon, L. M. (2003). The insula (Island of Reil) and its role in auditory processing. Literature review. Brain Research Reviews, 42(2), 143-154.

Birn, R. M., Diamond, J. B., Smith, M. A., \& Bandettini, P. A. (2006). Separating respiratory-variation-related fluctuations from neuronal-activity-related fluctuations in fMRI. Neuroimage, 31(4), 1536-1548.

Biswal, B., Yetkin, F. Z., Haughton, V. M., \& Hyde, J. S. (1995). Functional connectivity in the motor cortex of resting human brain using echo-planar MRI. Magnetic Resonance, 34(4), 537-541.

Breitenstein, C., Jansen, A., Deppe, M., Foerster, A. F., Sommer, J., Wolbers, T., et al (2005). Hippocampus activity differentiates good from poor learners of a novel lexicon. Neuroimage, 25(3), 958-968.

Breitenstein, C., \& Knecht, S. (2002). Development and validation of a language learning model for behavioral and functional-imaging studies. Journal of Neuroscience Methods, 114(2), 173-179.

Büchel, C., Coull, J. T., \& Friston, K. J. (1999). The predictive value of changes in effective connectivity for human learning. Science, 283(5407), 1538-1541.

Buchsbaum, B. R., \& D'Esposito, M. D. (2008). The search for the phonological store: From loop to convolution. Journal of Cognitive Neuroscience, 20(5), 762-778.

Chee, M. W., Soon, C. S., Lee, H. L., \& Pallier, C. (2004). Left insula activation: A marker for language attainment in bilinguals. Proceedings of the National Academy of Sciences USA, 101(42), 15265-15270.

Cherkassky, V. L., Kana, R. K., Keller, T. A., \& Just, M. A. (2006). Functional connectivity in a baseline resting-state network in autism. Neuroreport, 17(16), 1687-1690.

Clark, D., \& Wagner, A. D. (2003). Assembling and encoding word representations: fMRI subsequent memory effects implicate a role for phonological control. Neuropsychologia, 41(3), 304-317.

Cordes, D., Haughton, V. M., Arfanakis, K., Wendt, G. J., Turski, P. A., Moritz, C. H., et al. (2000). Mapping functionally related regions of brain with functional connectivity MR imaging. AJNR - American Journal of Neuroradiology, 21(9), 1636-1644.

Cornelissen, K., Laine, M., Renvall, K., Saarinen, T., Martin, N., \& Salmelin, R. (2004). Learning new names for new objects: Cortical effects as measured by magnetoencephalography. Brain and Language, 89(3), 617-622.

Damoiseaux, J. S., Rombouts, S. A., Barkhof, F., Scheltens, P., Stam, C. J., Smith, S. M., et al. (2006). Consistent resting-state networks across healthy subjects. Proceedings of the National Academic Science USA, 103(37), 13848-13853.

Davis, M. H., Di Betta, A. M., Macdonald, M. J. E., \& Gaskell, M. G. (2008). Learning and consolidation of novel spoken words. Journal of Cognitive Neuroscience, 21(4), 803-820.

Dronkers, N. F. (1996). A new brain region for coordinating speech articulation. Nature, 384(6605), 159-161. 
Friston, K. J., Frith, C. D., Liddle, P. F., \& Frackowiak, R. S. (1993). Functiona connectivity: The principal-component analysis of large (PET) data sets. Journal of Cerebral Blood Flow and Metabolism, 13(1), 5-14.

Greicius, M. D., Krasnow, B., Reiss, A. L., \& Menon, V. (2003). Functional connectivity in the resting brain: A network analysis of the default mode hypothesis. Proceedings of the National Academic Science USA, 100(1), 253-258.

Gronholm, P., Rinne, J. O., Vorobyev, V., \& Laine, M. (2005). Naming of newly learned objects: A PET activation study. Cognitive Brain Research, 25(1), 359-371.

Gullberg, M., Indefrey, P. (2003). Language background questionnaire. Nijmegen: Max Planck Institute for Psycholinguistics. <http://www.mpi.nl/research/researchprojects/the-dynamics-of-multilingual-processing>.

Gullberg, M., Roberts, L., Dimroth, C. (in press). What word-level knowledge can adult learners acquire after minimal exposure to a new language? International Review of Applied Linguistics.

Hampson, M., Peterson, B. S., Skudlarski, P., Gatenby, J. C., \& Gore, J. C. (2002). Detection of functional connectivity using temporal correlations in MR images. Human Brain Mapping, 15(4), 247-262.

Lee, H., Devlin, J. T., Shakeshaft, C., Stewart, L. H., Brennan, A., Glensman, J., et al. (2007). Anatomical traces of vocabulary acquisition in the adolescent brain. Journal of Neuroscience, 27(5), 1184-1189.

Liang, M., Zhou, Y., Jiang, T., Liu, Z., Tian, L., Liu, H., et al. (2006). Widespread functional disconnectivity in schizophrenia with resting-state functional magnetic resonance imaging. Neuroreport, 17(2), 209-213.

Lowe, M. J., Mock, B. J., \& Sorenson, J. A. (1998). Functional connectivity in single and multislice echoplanar imaging using resting-state fluctuations. Neuroimage, $7(2), 119-132$

Macmillan, N. A., \& Creelman, C. D. (2005). Detection theory: A user's guide (2nd ed.). Mahwah, NJ: Lawrence Erlbaum Associates.

McIntosh, A. R., Rajah, M. N., \& Lobaugh, N. J. (1999). Interactions of prefronta cortex in relation to awareness in sensory learning. Science, 284(5419), 1531-1533.

McNealy, K., Mazziotta, J. C., \& Dapretto, M. (2006). Cracking the language code Neural mechanisms underlying speech parsing. Journal of Neuroscience, 26(29) 7629-7639.

Mei, L., Chen, C., Xue, G., He, Q., Li, T., Xue, F., et al. (2008). Neural predictors of auditory word learning. Neuroreport, 19(2), 215-219.

Mori, E., Yamadori, A., \& Furumoto, M. (1989). Left precentral gyrus and Broca's aphasia: a clinicopathologic study. Neurology, 39(1), 51-54.
Paulesu, E., Frith, C. D., \& Frackowiak, R. S. (1993). The neural correlates of the verbal component of working memory. Nature, 362(6418), 342-345.

Raboyeau, G., Marie, N., Balduyck, S., Gros, H., Demonet, J. F., \& Cardebat, D. (2004). Lexical learning of the English language: A PET study in healthy French subjects. Neuroimage, 22(4), 1808-1818.

Raichle, M. E., MacLeod, A. M., Snyder, A. Z., Powers, W. J., Gusnard, D. A., \& Shulman, G. L. (2001). A default mode of brain function. Proceedings of the National Academic Science USA, 98(2), 676-682.

Saffran, J. R., Aslin, R. N., \& Newport, E. L. (1996). Statistical learning by 8-monthold infants. Science, 274, 1926-1928.

Salvador, R., Suckling, J., Coleman, M. R., Pickard, J. D., Menon, D., \& Bullmore, E. (2005). Neurophysiological architecture of functional magnetic resonance images of human brain. Cerebral Cortex, 15(9), 1332-1342.

Smith, E. E., Jonides, J., Marshuetz, C., \& Koeppe, R. A. (1998). Components of verbal working memory: Evidence from neuroimaging. Proceedings of the National Academic Science USA, 95(3), 876-882.

Stark, D. E., Margulies, D. S., Shehzad, Z. E., Reiss, P., Kelly, A. M. C., \& Uddin, L. Q. (2008). Regional variation in interhemispheric coordination of intrinsic hemodynamic fluctuations. Journal of Neuroscience, 28(51), 13754-13764.

Tian, L., Jiang, T., Wang, Y., Zang, Y., He, Y., Liang, M., et al. (2006). Altered restingstate functional connectivity patterns of anterior cingulate cortex in adolescents with attention deficit hyperactivity disorder. Neuroscience Letters, 400(1-2), 39-43.

Toni, I., Rowe, J., Stephan, K. E., \& Passingham, R. E. (2002). Changes of corticostriatal effective connectivity during visuomotor learning. Cerebral Cortex, 12(10), 1040-1047.

Tonkonogy, J., \& Goodglass, H. (1981). Language function, foot of the third frontal gyrus, and rolandic operculum. Archives of Neurology, 38(8), 486-490.

Tzourio-Mazoyer, N., Landeau, B., Papathanassiou, D., Crivello, F., Etard, O., Delcroix N., et al. (2002). Automated anatomical labeling of activations in SPM using a macroscopic anatomical parcellation of the MNI MRI single-subject brain. Neuroimage, 15(1), 273-289.

Waites, A. B., Stanislavsky, A., Abbott, D. F., \& Jackson, G. D. (2005). Effect of prior cognitive state on resting state networks measured with functional connectivity. Human Brain Mapping, 24(1), 59-68.

Wong, P. C., Perrachione, T. K., \& Parrish, T. B. (2007). Neural characteristics of successful and less successful speech and word learning in adults. Human Brain Mapping, 28(10), 995-1006. 